\title{
Influence of Pyrophosphate on the Interaction of Leuconostoc Phosphoglycerate Mutase with Cibacron Blue
}

\author{
Keiichi KawaI, Kazuhisa KumagaI, Mitsuko MoRI \\ and Hiroyuki HoRITSU \\ Department of Agricultural Chemistry, Faculty of Agriculture, \\ Gifu University, Gifu, Gifu 501-11, Japan
}

Received June 3, 1982

\begin{abstract}
Phosphoglycerate mutase (EC 2.7.5.3) of Leuconostoc dextranicum AHU 1078 adsorbed onto blue dextran-Sepharose, was easily eluted with a low concentration of pyrophosphate. Pyrophosphate inhibited the mutase competitively with respect to 3-phosphoglycerate, but noncompetitively with respect to 2,3-bisphosphoglycerate. Further inhibition studies suggested that two molecules of pyrophosphate bound per active site of the phosphoglycerate mutase. The inactivation of the Leuconostoc mutase by pyridoxal phosphate was prevented by the addition of pyrophosphate. Spectral measurements revealed that the mutase-Cibacron Blue complex in solution was dissociated by the addition of pyrophosphate.
\end{abstract}

Rabbit muscle phosphoglycerate mutase has been shown to be adsorbed to blue dextranSepharose and to be eluted with $0.5 \mathrm{~mm} 2,3-$ bisphosphoglycerate. ${ }^{1,2)}$

In a previous study ${ }^{3)}$ we found that phosphoglycerate mutase of Leuconostoc dextranicum could be eluted by a low concentration of 3-phosphoglycerate as well as 2,3-bisphosphoglycerate from blue dextran-Sepharose. Furthermore, several phosphorylated compounds such as ATP, ADP, GTP, GDP and $\operatorname{NADP}(\mathrm{H})$ also eluted the mutase. This elution behaviour indicates that the phosphate moiety of the phosphorylated compounds plays a significant role in eluting the mutase from blue dextran-Sepharose.

Recently we found that pyrophosphate was capable of eluting the Leuconostoc mutase from blue dextran-Sepharose. To elucidate the role of the phosphate moiety in the phosphorylated compounds in eluting the mutase, we examined the influence of pyrophosphate on the interaction of the mutase with Cibacron Blue.

\section{MATERIALS AND METHODS}

Microorganism. Leuconostoc dextranicum AHU 1078 was used throughout this study. The cultivation and preparation of washed cells was as described previously. ${ }^{4)}$

Enzyme. The crude extract was prepared as described previously. ${ }^{3)}$ The Leuconostoc mutase was purified by affinity chromatography using blue dextran-Sepharose. ${ }^{3)}$ The purified enzyme was apparently homogeneous on $7 \%$ polyacrylamide gel disc electrophoresis.

Enzyme assay. The activity of phosphoglycerate mutase was determined by the method of Grisolia and Carreras. ${ }^{5}$ )

Protein content. Protein content was measured according to the method of Folin and Ciocalteu. ${ }^{6)}$

Chemicals. Blue dextran-Sepharose 4B was prepared by the method of Ryan and Vestling. ${ }^{7)}$ The blue dextranSepharose used in this study had about $10 \mathrm{mg}$ of blue dextran per $\mathrm{ml}$ of packed Sepharose. 3-Phosphoglycerate and 2,3-bisphosphoglycerate were obtained from Boehringer Mannheim $\mathrm{GmbH}$, West Germany. Yeast enolase was a product of Oriental Yeast Co., Ltd., Tokyo. Cibacron Blue F3GA was obtained from Polysciences Inc. The blue dye was purified by the method of Weber et al. ${ }^{8)}$

\section{RESULTS}

Elution of phosphoglycerate mutase from blue dextran-Sepharose with orthophosphate or pyrophosphate

A portion of a cell-free crude extract $(82.8$ units, $6 \mathrm{ml}$ ) of Leuc. dextranicum was applied 
onto a column $(2 \times 10 \mathrm{~cm})$ of blue dextranSepharose. After the column had been washed with $0.1 \mathrm{M}$ Tris- $\mathrm{HCl}$ buffer ( $\mathrm{pH} 7.5)$ extensively, it was tested whether the enzyme was eluted with orthophosphate or pyrophosphate. At a concentration of $1 \mathrm{~mm}$, orthophosphate eluted the mutase with only a $13.3 \%$ yield, but pyrophosphate was quite good $(94.4 \%)$ for eluting the mutase from blue dextran-Sepharose. Furthermore, the mutase was eluted as a sharp peak with a linear gradient of pyrophosphate between 0 and $0.5 \mathrm{~mm}$. The

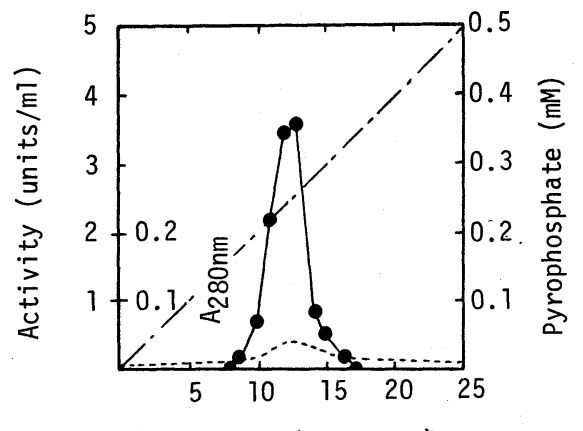

Fraction No. ( $5 \mathrm{ml} /$ tube)

Fig. 1. Elution Pattern of Phosphoglycerate Mutase from Blue Dextran-Sepharose with a Linear Gradient of Pyrophosphate.

The flow rate was $15 \mathrm{ml}$ per hr.

- - , phosphoglycerate mutase; ------, absorbance at $280 \mathrm{~nm}$; ----, pyrophosphate concentration.

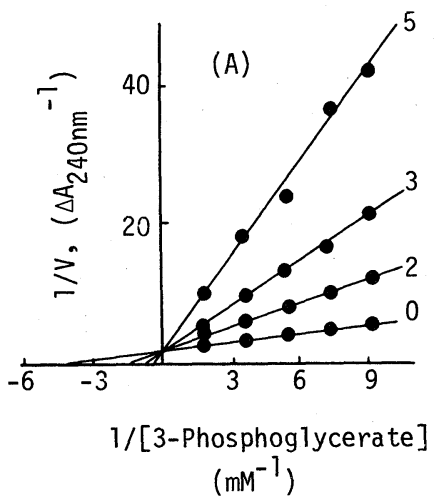

concentration of pyrophosphate for the peak fraction was $0.25 \mathrm{~mm}$ (Fig. 1). By this procedure the enzyme was purified 75.6-fold with a $57.6 \%$ yield.

Effect of pyrophosphate on the activity of phosphoglycerate mutase

As shown in Fig. 2, the inhibition by pyrophosphate with respect to 3-phosphoglycerate was found to be competitive and with respect to 2,3-bisphosphoglycerate to be noncompetitive. Furthermore, plots of the enzyme activity as a function of the pyrophosphate concentration are shown in Fig. 3(A). ${ }^{9)}$ At a high concentration of 3-phosphoglycerate, the plots gave a near straight line, but at a low concentration, the plots were found to fit a parabola. That is, replots of the reciprocal of the initial velocity against the square of the pyrophosphate concentration gave a linear curve as can be seen in Fig. 3(B). As shown in Fig. 4, the Hill's coefficient $(n)^{10,11)}$ was calculated to be about 2 at a low concentration of 3phosphoglycerate.

Protective effect of pyrophosphate on inactivation of phosphoglycerate mutase by pyridoxal phosphate

When the Leuconostoc phosphoglycerate mutase was incubated with pyridoxal phos-

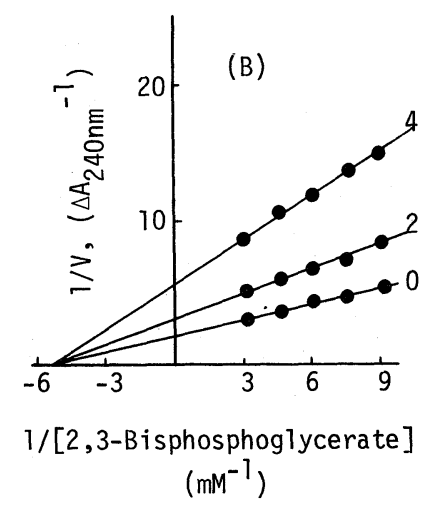

FIG. 2. Plots of the Initial Velocity against the Reciprocal of the Concentration of 3-Phosphoglycerate (A) or 2,3-Bisphosphoglycerate (B) in the Absence and Presence of Pyrophosphate.

The numbers in the figure represent pyrophosphate concentration (mM).

(A) The concentration of 2,3-bisphosphoglycerate was held constant at $0.33 \mathrm{~mm}$.

(B) The concentration of 3-phosphoglycerate was held constant at $16.7 \mathrm{~mm}$. 

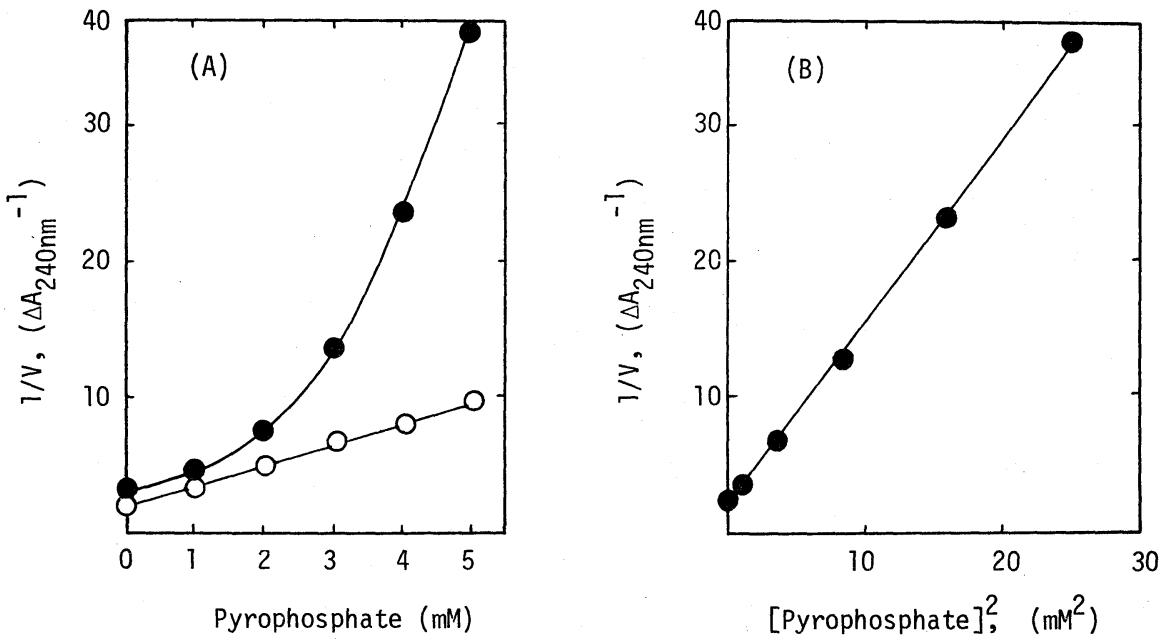

Fig. 3. (A) Plots of the Reciprocal of the Initial Velocity with Respect to Pyrophosphate Concentration. The concentrations of 3-phosphoglycerate were held constant at $0.56 \mathrm{~mm}(\mathcal{O})$ or $1.67 \mathrm{~mm}(\mathrm{O})$. The concentration of 2,3-bisphosphoglycerate was held constant at $0.33 \mathrm{~mm}$.

(B) Replots of the Reciprocal of the Initial Velocity against the Square of the Pyrophosphate Concentration. The data from Fig. 3 (A) were replotted.

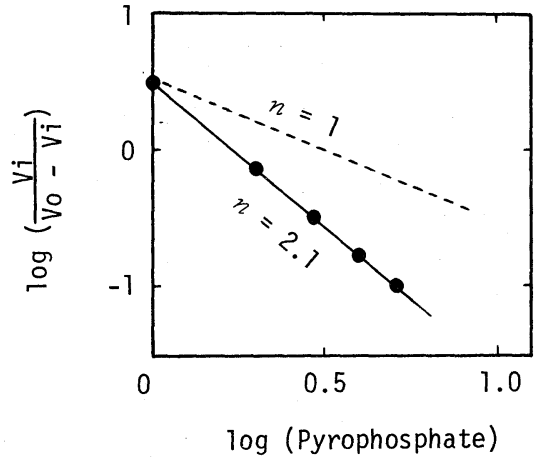

FIG. 4. Phosphoglycerate Mutase Activity as a Function of Pyrophosphate Concentration.

The concentrations of 3-phosphoglycerate and 2,3-bisphosphoglycerate were held constant at $0.56 \mathrm{~mm}$ and $0.33 \mathrm{~mm}$, respectively.

phate, the mutase was rapidly inactivated resulting in the loss of about $60 \%$ of the original activity. This inactivation was prevented by the presence of either 3-phosphoglycerate or blue dextran. ${ }^{3)}$ As shown in Table I, pyrophosphate also protected the phosphoglycerate mutase from the inactivation by pyridoxal phosphate.

\section{Difference spectroscopy studies}

In a previous study, ${ }^{3)}$ we found that the
Table I. Protective Effect of PyRoPHOSPHATE ON THE INACTIVATION of Phosphoglycerate Mutase By Pyridoxal Phosphate

\begin{tabular}{lcc}
\hline & \multicolumn{2}{c}{ Remaining activity $(\%)$} \\
\cline { 2 - 3 } \multicolumn{1}{c}{ Addition } & \multicolumn{2}{c}{ Incubation time (min) } \\
\cline { 2 - 3 } & 5 & 10 \\
\hline None & 95 & 97 \\
$\begin{array}{l}\text { Pyridoxal phosphate }(0.1 \mathrm{mM}) \\
\text { Pyridoxal phosphate }(0.1 \mathrm{mM})\end{array}$ & 43 & 38 \\
plus pyrophosphate $(1 \mathrm{~mm})$ & 92 & 80 \\
\hline
\end{tabular}

extinction at $680 \mathrm{~nm}$ of the difference spectrum produced by the titration of the Leuconostoc phosphoglycerate mutase with Cibacron Blue was decreased progressively by the addition of 3-phosphoglycerate. Addition of pyrophosphate also resulted in a progressive decrease in the extinction. Thus, the enzyme-dye complex was dissociated by the addition of pyrophosphate.

\section{DISCUSSION}

Pyrophosphate at a low concentration was found to elute the Leuconostoc phosphoglyc- 
erate mutase from blue dextran-Sepharose. This suggests that the electrostatic interaction has a main role in binding of the mutase to blue dextran-Sepharose.

Pyrophosphate inhibited the phosphoglycerate mutase competitively with respect to 3phosphoglycerate and noncompetitively with respect to 2,3-bisphosphoglycerate. Further inhibition studies indicated that two molecules of pyrophosphate bind per active site of the mutase at a low concentration of 3-phosphoglycerate. Leuconostoc phosphoglycerate mutase was composed of two identical subunits having a molecular weight of 28,000 . It is likely that one molecule of pyrophosphate binds to the 3-phosphoglycerate-binding site of each subunit.

The inactivation of the Leuconostoc phosphoglycerate mutase by pyridoxal phosphate was prevented by the presence of either pyrophosphate or 3-phosphoglycerate. This result indicates that pyridoxal phosphate binds to the 3-phosphoglycerate-binding site, resulting in inactivation of the mutase.

Since the Leuconostoc mutase-Cibacron Blue complex in solution was dissociated by the addition of pyrophosphate, the phosphate moiety of the phosphorylated compounds ca- pable of eluting the phosphoglycerate mutase from blue dextran-Sepharose may play an important role in eluting the mutase from blue dextran-Sepharose.

\section{REFERENCES}

1) E. Stellwagen, R. Cass, S. T. Thompson and M. Woody, Nature, 257, 716 (1975).

2) S. T. Thompson, K. H. Cass and E. Stellwagen, Proc. Natl. Acad. Sci., U.S.A., 72, 669 (1975).

3) K. Kawai, M. Mori, T. Eguchi, H. Horitsu and Y. Eguchi, Agric. Biol. Chem., 45, 2781 (1981).

4) S. Kakimoto, K. Kawai, S. Yashima and Y. Sasaki, J. Gen. Appl. Microbiol., 18, 1 (1972).

5) S. Grisolia and J. Carreras, "Methods in Enzymology," Vol. XLII, ed. by W. A. Wood, Academic Press Inc., New York and London, 1975, p. 435.

6) O. Folin and V. Ciocalteu, J. Biol. Chem., 73, 627 (1927).

7) L. D. Ryan and C. S. Vestling, Arch. Biochem. Biophys., 160, 279 (1974).

8) B. H. Weber, K. Willeford, J. G. Moe and D. Piszkiewicz, Biochem. Biophys. Res. Commun., 86, 252 (1979).

9) M. Dixon and E. Webb, "Enzymes," Longman Group Ltd., London, 1979, p. 332.

10) J. P. Changeux, Cold Spring Harbor Symposium, Quant. Biol., 28, 497 (1963).

11) J. Monod, J. P. Changeux and F. Jacob, J. Mol. Biol., 6, 306 (1963). 